\title{
Exopolyphosphatase of Lysinibacillus sphaericus is Essential for Stresses Resistance and Production of Binary Toxins
}

\author{
Tingyu Shi ${ }^{1,2}$, Xinggao Dong ${ }^{1}$, Yimin $\mathrm{Hu}^{2}$, Xiaomin $\mathrm{Hu}^{2}$, Zhiming Yuan ${ }^{2 *}$ \\ ${ }^{1}$ Department of Basic Medicine, Medical School of Hubei Institute for Nationalities, Enshi 445000, People's Republic of China \\ ${ }^{2}$ Key Laboratory of Agricultural and Environmental Microbiology, Wuhan Institute of Virology, Chinese Academy of Sciences, Wuhan 430071, People's \\ Republic of China
}

Received: April 08, 2016; Accepted: May 04, 2016; Published: May 07, 2016

*Corresponding author: Zhiming Yuan, Wuhan Institute of Virology, Chinese Academy of Sciences, Wuhan 430071, China, Tel: +86 27 87198195; Fax: + $862787199480 ;$ E-mail: yzm@wh.iov.cn

\begin{abstract}
The Exopolyphosphatase (PPX) of Lysinibacillus sphaericus is encoded by the Bsph_1303 gene (ppx), being responsible for the hydrolysis of inorganic polyphosphates to orthophosphate (Pi). In the present work, we identified and characterized the ppx from $L$. sphaericus. We found that ppx disruption resulted in massive polyP accumulation, compromised the resistance to high osmotic, high $\mathrm{pH}$ and high temperature, and impaired the motility and biofilm formation. Additionally, results obtained from the ppx null mutant demonstrated that PPX was involved in the production of binary toxins and growth in MBS medium, a medium for toxicity production. These result indicated that PPX of $L$. sphaericus appeared to be essential for the resistance to stresses and binary toxins production.
\end{abstract}

Keywords: Polyphosphate; Binary toxins; Lysinibacillus sphaericus; Exopolyphosphatase

\section{Abbreviations}

PolyP: Polyphosphate; PPX: Exopolyphosphatase

\section{Introduction}

Inorganic Polyphosphates (polyPs) are linear polymers consisting of tens to hundreds of Orthophosphate (Pi) residues linked by energy-rich phosphoanhydride bonds. Numerous investigations indicate that polyP is essential for the growth of microorganisms, their responses to various environmental stresses, and the virulence of pathogens [1]. PolyP is synthesized by Polyphosphate Kinase (PPK) that catalyses the reversible transfer of terminal phosphate of ATP to polyP chain [2]. PolyP can be degraded by the Exopolyphosphatase (PPX) that releases Pi processively from the termini of the polyP chain [3].

Although polyP has been shown to have important functions in many microorganisms, there are few studies on the role of this polymer in Bacillus. Feasible reason is that the important model organism, Bacillus subtilis does not harbor the ppk gene [1]. The first study about polyP in Bacillus cereus demonstrated that polyp is involved in motility, biofilm formation, and sporulation
[4]. Other investigations reported that the exogenous longchain polyP inhibited the cereulide toxin synthesis [5] and septum formation, and caused cell lysis [6]. Additionally, in Bacillus thuringiensis, overexpression of ppk gene increases bioinsecticide production [7].

L. sphaericus is mesophilic, aerobic rod-shaped bacteria, and some of them produce parasporal crystal proteins specifically toxic to Culex and Anopheles mosquito larvae and have been widely used as biocontrol agents. The larvicidal activity of $L$. sphaericus is mainly due to the production of two crystalline mosquito-larvicidal proteins of $42 \mathrm{kDa}$ (BinA) and $51 \mathrm{kDa}$ (BinB) during sporulation $[8,9]$. L. sphaericus is considered one of the most successful microbial larvicide and has been commercialized over the past decade [10]. Besides the binary toxins, some other toxins such as sphaericolysin, vegetative mosquitocidal toxins, Cry48/ Cry49 toxin are also responsible for its larvicidal activity [10]. To date, the mechanism by which Bin kills mosquito larvae remains largely unsolved. One hypothesis is that pore formation by binary toxins may be sensed by p38 mitogen-activated protein kinase, whose activity might induce autophagy $[10,11]$. Moreover, to our knowledge there was nearly no study on resistance of L. sphaericus to environmental stresses, which was essential for its recycling in mosquito breeding sites. Recently, we identified the ppk gene of L. sphaericus C3-41 and found that ppk gene affected polyP accumulation and bacterial growth [12]. Various stresses were encountered when L. sphaericus accessed to the haemocoel of mosquito and recycled in mosquito cadaver $[13,14]$. So, responses to all stresses were essential for growth and toxin production of $L$. sphaericus. Recent genetic and molecular analyses have revealed how several strategies enable bacteria to persist and overcome host immune defences [15]. PolyP is essential for growth, survival and the virulence of pathogens $[1,16]$.

Here, we have provided evidence that PPX of L. sphaericus is required for stress resistance such as osmotic pressure, high $\mathrm{pH}$ value and high temperature, and for motility and biofilm 
formation. We also demonstrated that binary toxins production is affected by PPX.

\section{Materials and Methods}

\section{Bacterial strains and plasmids}

The bacterial strains and plasmids used for this study are listed in Table 1. Escherichia coli DH5 $\alpha$ and BL21 (DE3) were used as hosts for cloning and expression, respectively. The ppx was amplified from $L$. sphaericus C3-41 genomic DNA with the primer pairs: Fppx and Rppx. The PCR product was excised and cloned between the BamHI/SacI sites of pET28a+ to generate the plasmid pET28a-BsPPX.

Null mutant allele of ppx was obtained by using a two-step PCR strategy [12]. The resulting plasmid for allelic displacement and reverse mutation of ppx were named as pNR5101-PPX and pHT304-ppx, respectively. The primers were listed in Table 2.

\section{Media and growth conditions}

Unless otherwise specified, E. coli strain was grown at $37^{\circ} \mathrm{C}$ in Luria-Bertani (LB) medium. L. sphaericus were grown at $30^{\circ} \mathrm{C}$ in LB or MBS medium ( $\mathrm{pH}$ 7.4) [17]. Antibiotics were added to growth media at the following final concentrations: Ampicillin $(100 \mu \mathrm{g} / \mathrm{ml})$, Erythromycin $(20 \mu \mathrm{g} / \mathrm{ml})$, Kanamycin $(50 \mu \mathrm{g} / \mathrm{ml}$ for E. coli, $10 \mu \mathrm{g} / \mathrm{ml}$ for L. sphaericus) and tetracycline (10 $\mu \mathrm{g} /$ $\mathrm{ml}$ ). For solid medium, agar was added to a final concentration of $1.5 \%$ (w/v).

\section{Cloning and expression of ppx genes}

Recombinant plasmid pET28a-PPX was transformed into $E$.

Table 1: Strains and plasmids used for this study.

\begin{tabular}{|l|l|l|}
\hline Strains or plasmids & Characteristics & Source or reference \\
\hline Escherichia coli & & \\
\hline JM109 & Cloning host & Lab stock \\
\hline BL21(DE3) & Expression host & Lab stock \\
\hline B. sphaericus & & \\
\hline C3-41 & Wild type strain bearing native pBsph plasmid & Lab stock \\
\hline$\Delta p p x$ & C3-41 mutant with deletion of the $p p x$ gene & This study \\
\hline$\Delta p p x$ (pHT304-ppx) & Appx with plamid pHT304-ppx & This study \\
\hline Plasmids & & \\
\hline pET28a & Expression vector in $E$. coli & Lab stock \\
\hline pET28a-PPX & pET28a with $p p x$ gene & This study \\
\hline pRN5101 & Recombinant vector used in gene disruption experiments, Amp ${ }^{\mathrm{r}}$, Erm ${ }^{\mathrm{r}}$ & [26] \\
\hline pRN5101-PPX & pRN5101 carrying partial $p p x$ deletion gene & This study \\
\hline pHT304 & Plasmid designed for gene expression in Bacillus. & {$[27]$} \\
\hline pHT304-spc & pHT304 with $s p c$ promoter region & This study \\
\hline pHT304-ppx & pHT304 with $p p x$ gene fused to the downstream of $s p c$ promoter region & This study \\
\hline pMarC333 & Source of the spectinomycin promoter region & {$[28]$} \\
\hline
\end{tabular}

Table 2: Oligonucleotide primers designed to generate DNA fragment for plasmid construction.

\begin{tabular}{|c|c|c|}
\hline Primers & Sequences (5' to $3^{\prime}$ ) & Restriction sites \\
\hline \multicolumn{3}{|c|}{ Primers used in plasmid construction } \\
\hline Fppx & CGCGGATCCATGCTTGAAGGATGTGATTATC & BamHI \\
\hline Rppx & TA CGAGCTCGGCTCATTATTCGTTATTT & SacI \\
\hline Fppx-up-ko & ACGCGTCGACTGTTCCATGTGGATAACGTGACA & SalI \\
\hline Rppx-up-ko & GCCССТCTTCATTGTTATACTGATA & \\
\hline Fppx-down-ko & AAGGATGGAATAAGGATGACAAC & \\
\hline Rppx-down-ko & CTAGCTAGCCCTCCAGTAGTACAACGACTGTT & Nhe I \\
\hline Fkan-ppx & TATCAGTATAACAATGAAGAGGGGCTAAACCCAGCGAACCA & \\
\hline Rkan-ppx & GTTGTCATCCTTATTCCATCCTTTCTAGGTACTAAAACA & \\
\hline Fppx-up & TGTGAACAAAATAAAC & \\
\hline Rppx-up & TGGTTCGCTGGGTTTA & \\
\hline Fppx-down & GTCATTGGGATTTTTA & \\
\hline Rppx-down & TGTTTTAGTACCTAGA & \\
\hline Fppx-com & CGCGGATCCATGCTTGAAGGATGTGATTATC & BamHI \\
\hline Rppx-com & TA CGAGCTCGGCTCATTATTCGTTATTT & SacI \\
\hline Fspc-promoter & CCCAAGCTTAGAGTTGGTAGCTCTTGATCC & HindIII \\
\hline Rspc-promoter & CGCGGATCCGATTTCACCTCGTTGATTATGT & BamHI \\
\hline
\end{tabular}


coli BL21 (DE3). Cells were grown at $37^{\circ} \mathrm{C}$ to an OD600 of 0.6 . For expression of PPX, IPTG was added to a final concentration of $0.1 \mathrm{mM}$ and growth was continued for $16 \mathrm{~h}$ at $20^{\circ} \mathrm{C}$. Cells were harvested, re-suspended in $10 \mathrm{mM}$ Tris- $\mathrm{HCl}$ (pH 7.5) with $1 \mathrm{mM}$ $\mathrm{MgCl}_{2}, 1 \mathrm{mM}$ PMSF and disrupted by sonication. Recombinant and His6-tagged PPX was purified from the cell-free supernatant by chromatography on Ni2+-NTA agarose.

\section{PPX activity assay}

The activity of PPX was measured by the detection of Pi product [3]. Briefly, the reaction mixture $(100 \mu \mathrm{L})$ contained $50 \mathrm{mM}$ Tricine/ KOH (pH 8.0), 1 mM $\mathrm{MgCl}_{2}, 175 \mathrm{mM} \mathrm{KCl}, 22 \mu \mathrm{g}$ PPX, and polyP as indicated (provided kindly by Shinichi Kato [Regene Tiss Inc.]). $\mathrm{MgCl}_{2}$ was added last to avoid precipitation of the polyP. The Pi generated by PPX was continuously assayed for 45 min using Molecular Probes' EnzChek ${ }^{\circledR}$ Phosphate Assay Kit according to the manufacturer's protocol. One unit of enzyme is defined as the amount releasing $1 \mathrm{pmol}$ of phosphate from polyP per min.

Construction of ppx mutants pNR5101-PPX plasmid for knockout of ppx genes was transformed into L. sphaericus C3-41 by electroporation [12]. The resulting mutation of ppx gene was named as $\Delta \mathrm{ppx}$ and the reverse mutation of ppx gene was named as $\Delta \mathrm{ppxC}$. All primers for knockout and validation of $\Delta \mathrm{ppx}$ and construction reverse mutation of ppx were presented in Table 2 .

\section{Extraction and quantification of polyP}

The PolyP was extracted from L. sphaericus cells with Glass milk as described previously [18]. Briefly, $2 \mathrm{ml}$ of L. sphaericus WT, $\Delta$ ppx and $\Delta$ ppxC cultures at time points indicated were pelleted in a $1.5 \mathrm{ml}$ tube for $2 \mathrm{~min}$, respectively. To the pellet were added $0.5 \mathrm{ml}$ of $4 \mathrm{M}$ Guanidine Isothiocyanate (GITC)-50 mM Tris-HCl, pH 7.0 (GITC lysis buffer) and sonicated briefly; a $10 \mu \mathrm{L}$ sample was removed for protein estimation. To each tube were added $30 \mu \mathrm{L}$ of $10 \%$ sodium dodecyl sulphate, $0.5 \mathrm{ml}$ of $95 \%$ ethanol, and $5 \mu \mathrm{L}$ of Glass milk (Bio 101). After being centrifuged briefly, the pellet was then suspended in $0.5 \mathrm{ml}$ of cold New Wash buffer ( $5 \mathrm{mM}$ Tris- $\mathrm{HCl}$ [pH 7.5], $50 \mathrm{mM} \mathrm{NaCl}, 5 \mathrm{mM}$ EDTA and $50 \%$ ethanol). The washed pellet was then resuspended in $50 \mu \mathrm{L}$ of 50 $\mathrm{mM}$ Tris- $\mathrm{HCl}(\mathrm{pH} 7.4)-10 \mathrm{mM} \mathrm{MgCl}-20 \mu \mathrm{g}$ each of DNase I and RNase A per ml and incubated at $37^{\circ} \mathrm{C}$ for $10 \mathrm{~min}$ to remove DNA and RNA. The pellet was washed first with $150 \mu \mathrm{L}$ of 4 M GITC lysis buffer and $150 \mu \mathrm{L}$ of $95 \%$ ethanol, and then twice in New Wash buffer. PolyP was eluted from the pellet with $50 \mu \mathrm{L}$ of 50 $\mathrm{mM}$ Tris- $\mathrm{HCl}(\mathrm{pH} 8.0)$ at $95^{\circ} \mathrm{C}$ for $2 \mathrm{~min}$.

The amount of PolyP in L. sphaericus cells was quantitated as described previously [12]. The amount of polyP in $L$. sphaericus cells was measured based on the character that polyp can be transformed to ATP by PPK reverse activity. The ATP concentration was assayed with Bioluminescence Assay Kit CLSII (Roche), and concentration of polyp is given in terms of $\mathrm{Pi}$ residues.

\section{Resistance to stress conditions}

The resistance to stress conditions of wild type, $\Delta \mathrm{ppx}$ and $\Delta \mathrm{ppxC}$ were performed as described [19]. To test resistance to oxidative stress, $100 \mu \mathrm{L}$ overnight cultures were spread on LB agar medium in 90-mm-diameter Petri dishes. Subsequently, $3 \mathrm{MM}$ filter paper disc ( $5 \mathrm{~mm}$ in diameter) containing $5 \mu \mathrm{l}$ of a solution of $50 \mathrm{mM}$ menadione (sigma) were layered on the plates. After 2 days of incubation at $30^{\circ} \mathrm{C}$, the halos of growth inhibition were measured.

\section{Biofilm and motility assays}

Biofilm formation and motility assays were performed as described $[4,20]$.

\section{Western blot analysis}

L. sphaericus cultures were grown in MBS medium at $30^{\circ} \mathrm{C}$. Cells were harvested at indicated times and washed twice with sterile water and then centrifuged. The soluble proteins were electrophoresed in 12\% SDS-PAGE gels and subsequently transferred to PVDF membranes. After blocking, the membranes were incubated for 2 hours with primary antibody (anti-BinA, 1:3000 and anti-BinB 1:1000) followed by incubation with goat anti-mice HRP (horseradish peroxidase, 1:3000 dilution) secondary antibody (Millipore). Finally, membranes were developed using the luminal (ECL; Amersham Biosciences).

\section{Bioassays}

The toxicities of L. sphaericus C3-41, $\Delta \mathrm{ppx}$ and $\Delta \mathrm{ppxC}$ against fourth instar larvae of a susceptible Culex quinquefasciatus colony were assayed by bioassay, performed as described by Yang et al. [21]. Lethal concentrations of $50 \%$ and $90 \%$ were determined by probit analysis with a program indicating mean and Standard Error (SE).

\section{Statistical analysis}

Results are indicated as means and the standard deviation $(n=3-8)$. The significances of the difference of the means in experiments carried out with wild type, $\Delta \mathrm{ppx}$ and $\Delta \mathrm{ppxC}$ were calculated using a Student t text.

\section{Results}

\section{Expression of ppx and activity assays}

The gene encoding putative counterparts of PPX has been identified in L. sphaericus (Bsph_1303). The putative PPX of L. sphaericus C3-41 was of 524 amino acid residues, with a calculated molecular masses of $57.64 \mathrm{kDa}$, and $44 \%$ identical to those of B. cereus E33L. The gene products were expressed as $\mathrm{N}$-terminal His-tagged proteins in E. coli and purified by affinity chromatography on Ni2+-NTA agarose (Figure 1A). As shown in Figure 1B, recombinant PPX demonstrated polyP typedependent activity. The polyP14, polyP60 and polyP130 were hydrolyzed by PPX with a specific activity of $6.48 \times 103,6.83$ $\times 103$ and $14.24 \times 103$ units/ mg protein, respectively (Figure 1D). Furthermore, recombinant protein PPX also demonstrated a polyP concentration-dependent activity (Figure 1C), hydrolyzing $3 \mu \mathrm{m}$ polyP130 and $10 \mu \mathrm{m}$ polyP130 with specific activity of 6.75 $\times 103$ and $23.3 \times 103$ units / mg protein, respectively (Figure 1D). These results confirmed the function of PPX in polyP metabolism. 
A

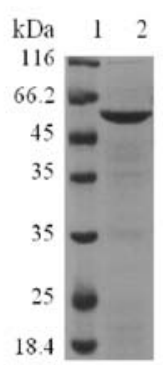

B

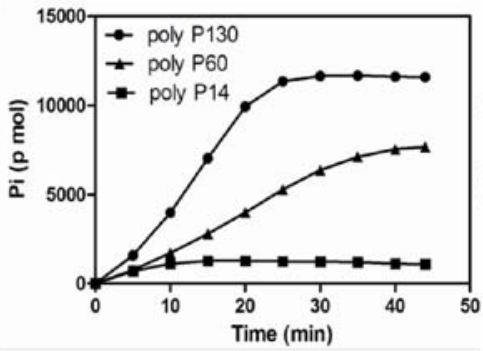

C

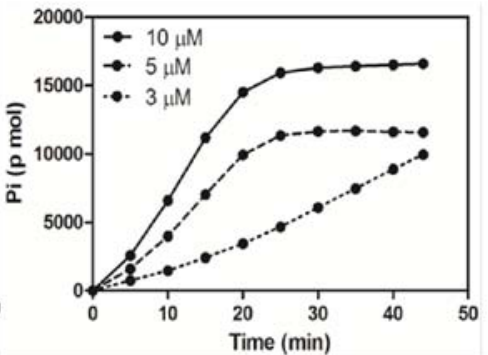

D

\begin{tabular}{cccccc}
\hline \multirow{2}{*}{ PolyP con. $(\mu \mathrm{M})$ and length } & $5 \mu \mathrm{M}$ & $5 \mu \mathrm{M}$ & $3 \mu \mathrm{M}$ & $5 \mu \mathrm{M}$ & $10 \mu \mathrm{M}$ \\
\cline { 2 - 6 } & PolyP14 & PolyP60 & PolyP 130 & PolyP 130 & PolyP 130 \\
\hline Specific Unit $\left(10^{3} \mathrm{U} / \mathrm{mg}\right)$ & 6.48 & 6.83 & 6.75 & 14.24 & 23.3 \\
\hline
\end{tabular}

Figure 1: Enzymatic activity of PPX. (A) SDS-PAGE of purified PPX. Lane 1: standard protein marker; lane 2: purified PPX; (B) Time course of different length polyP $(5 \mu \mathrm{m})$ degradation by PPX $(22 \mu \mathrm{g})$; (C) Time course of different concentration polyP130 degradation by PPX (22 $\mu \mathrm{g})$; (D) The specific activity of PPX when hydrolyzing different polyP length and concentration. The specific activity of PPX was measured according to (B) and (C).

\section{Inactivation of ppx resulting in polyP accumulation}

In order to prove the effect of the ppx gene on accumulation of polyP, the $\Delta \mathrm{ppx}$ was obtained by homologous recombination. As shown in Figure 2, there was significantly more abundant polyP in $\Delta$ ppx compared with that in WT and the polyP levels in $\Delta$ ppx were reduced significantly by expressing ppx gene.

\section{Effect of a mutation in ppx on the resistance to stresses in L. sphaericus}

L. sphaericus C3-41 WT and the $\Delta \mathrm{ppx}$ were grown in LB medium under different conditions (high salt, high temperature, low $\mathrm{pH}$, or high $\mathrm{pH}$ ) in order to evaluate their ability to grow under stress conditions (Figure 3). Growth under no stress conditions (LB medium) was not affected by PPX disruption (Figure 3A). The extent of the effect of the $\Delta$ ppx varied under the different stress conditions. The effect of PPX disruption seems different with stress. High salt seems little effect on initial growth rate but final OD. High $\mathrm{pH}$ seems to affect initial growth rate. High temperature seems not affect growing rate but decrease of OD seems earlier in $\Delta$ ppx mutant (Figure 3B, C and D). The defect could be partially restored by plasmid expression of ppx in the mutant strain. Growth in the presence of menadione (a superoxide radical generator) was not affected by the $\Delta \mathrm{ppx}$ under our experimental conditions (data not shown).

Additionally, the biofilm formation and swimming on semisolid agarose plates of mutants were dramatically decreased (Figure 4). It is clear that the decrease was not attributable to the impairment of growth, because the growth rates of mutants were indistinguishable from that of WT in LB medium (Figure 3A).

\section{Binary toxins production by mutant and control strains}

Growth of $\Delta$ ppx was impaired compared with WT when grown in MBS medium (Figure 5A), a medium for toxicity production [17]. In order to compare binary toxins production by $\Delta \mathrm{ppx}$ with that by WT, we analyzed protein levels of binary toxins in the bacterial cells with the same wet-weight through SDS-PAGE and

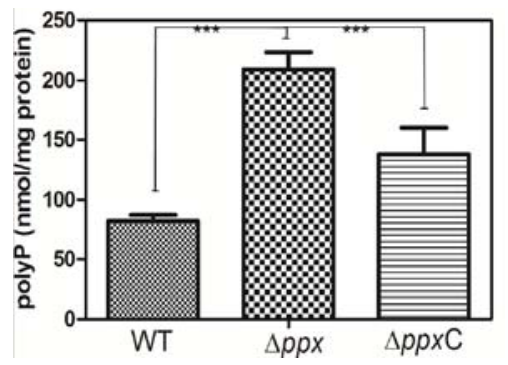

Figure 2: Measurement of polyP extracted from L. sphaericus WT, $\Delta \mathrm{ppx}$ and $\triangle \mathrm{ppxC}$ grown in LB to log-phase. The results are from three measurements. ${ }^{* * *}, \mathrm{p}<0.01$.
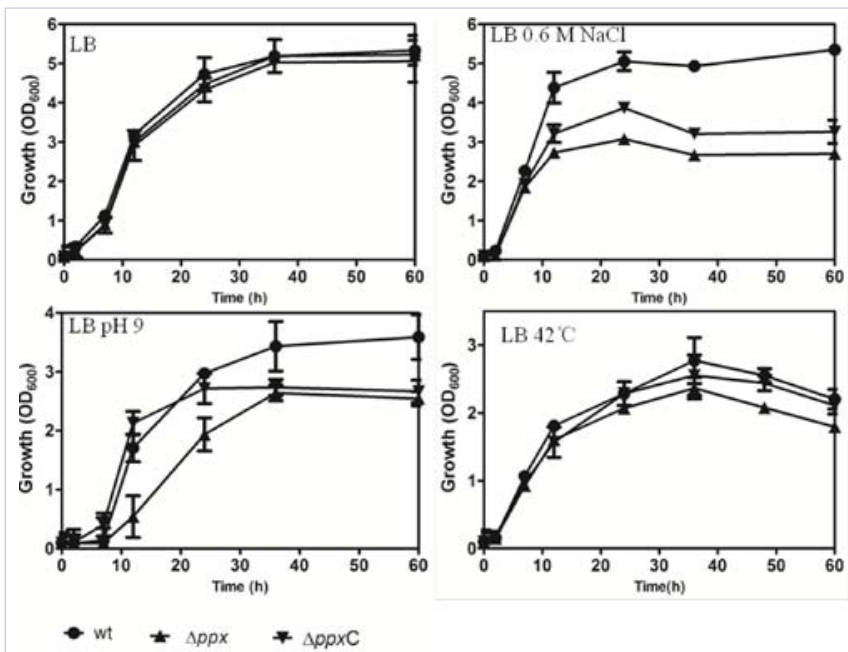

Figure 3: Motility and biofilm formation of WT and mutant. (A) Motility. Cells were inoculated on swimming plates with 12 -h incubation at $30^{\circ} \mathrm{C}$ and they were photographed. Each is representative of the strain; (B) Biofilm formation. An overnight LB culture $(2 \mu \mathrm{l})$ was inoculated into $200 \mu \mathrm{L}$ of $\mathrm{LB}$ in 96 -well plates and incubated at $30^{\circ} \mathrm{C}$ without shaking. After 12-h incubation, the media were removed; each well was rinsed with sterile water, and were stained with crystal violet, extracted and measured. Data represent mean values and standard deviations for eight replicates. ${ }^{* * *}, \mathrm{p}<0.01$. 
western blot. Results suggested that binary toxins production by the $\Delta \mathrm{ppx}$ was significant lower compared with WT at different times (Figure 5B and C). Bioassay results against fourth instar larvae showed that the toxicity of $\Delta \mathrm{ppx}$ was significant lower than that of wild type (Table 3). The binary toxins production and toxicity could be partially restored by expression of ppx gene in the mutant strain.

\section{Discussion}

In the present study, we identified the PPX function of $L$. sphaericus C3-41 and disrupted the ppx gene with homologous recombination. PolyP extraction and quantitation showed that polyP were significantly more abundant in $\Delta \mathrm{ppx}$ than in WT. Moreover, our data indicated that a high intracellular concentration of polyP in the $\Delta$ ppx compromised the resistance of L. sphaericus to high osmotic stress, high $\mathrm{pH}$ and high temperature,

\section{A}
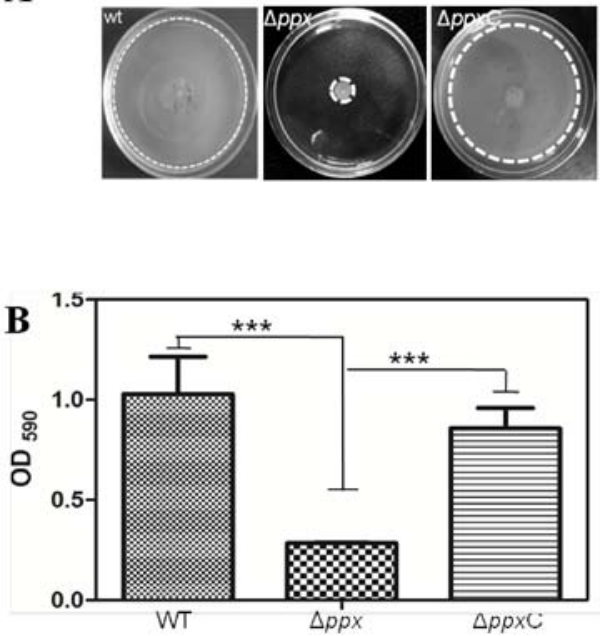

Figure 4: Effect of a mutant in $p p x$ from L. sphaericus on growth under different stress conditions. Growth curves of $L$. sphaericus strains (WT, $\Delta p p x$ and $\triangle p p x \mathrm{C}$ are shown. (A) Growth in LB medium; (B) growth in LB medium with $0.6 \mathrm{M} \mathrm{NaCl}$; (C) growth in $\mathrm{LB}$ medium adjusted to $\mathrm{pH}$ 9; (D) growth in $\mathrm{LB}$ medium at $42^{\circ} \mathrm{C}$. The results are the means of at least three experiments.

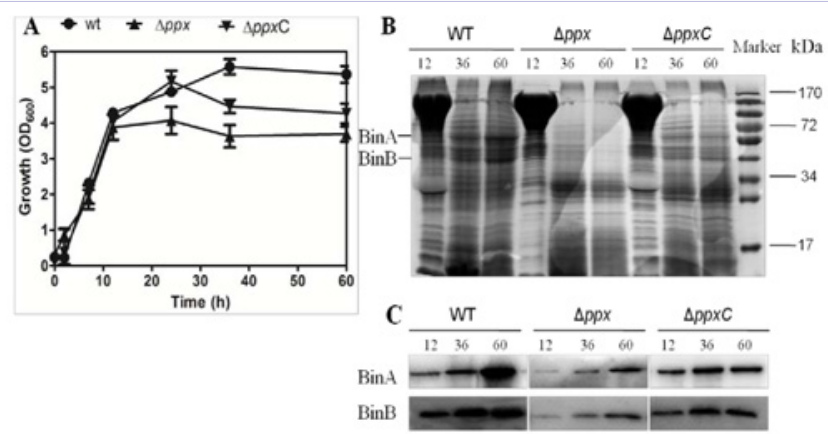

Figure 5: Effect of a mutant in ppx from L. sphaericus on growth and binary toxins production grown in MBS medium. (A) Growth curves of WT, $\Delta p p x$ and $\Delta p p x C$; (B) western blot analysis of binary toxins proteins synthesis WT, $\Delta p p x$ and $\Delta p p x \mathrm{C}$.
Table 3: Larvicidal activity of L. sphaericus C3-41, $\Delta p p x$ and $\Delta p p x \mathrm{C}$.

\begin{tabular}{|c|c|c|}
\hline Strains & $\mathrm{LC}_{50} *(95 \% \mathrm{CL})^{\dagger}$ & $\mathrm{LC}_{90} *(95 \% \mathrm{CL})^{\dagger}$ \\
\hline C3-41 & $\begin{array}{l}0.320(1.154<C L< \\
2.043) \dagger\end{array}$ & $\begin{array}{l}5.218(3.797<\mathrm{CL}< \\
8.548) \dagger\end{array}$ \\
\hline$\Delta p p x$ & $\begin{array}{l}9.544(16.021<\mathrm{CL}< \\
28.601) \dagger\end{array}$ & $\begin{array}{l}36.459(25.364<\mathrm{CL}< \\
71.270)\end{array}$ \\
\hline$\triangle p p x C$ & $\begin{array}{l}2.339(3.251<\mathrm{CL}< \\
4.438) \dagger\end{array}$ & $\begin{array}{l}7.224(9.111<\mathrm{CL}< \\
12.602) \dagger\end{array}$ \\
\hline
\end{tabular}

and impaired mobility and biofilm formation. Biofilm formation and motility is one of ways to adapt to stresses and stringencies by concentrating nutrients and escaping environmental stresses [22]. Previous study showed that ppk gene knockout resulted in polyP deficiency and affected the growth of L. sphaericus C341[12]. These results suggested that maintenance of the polyP levels within a normal range were essential for growth and its responses to stresses.

The Binary toxins comprises two proteins, BinA and BinB that are co-transcribed from a single operon just before the end of exponential growth and into sporulation, and strains blocked in early stage sporulation do not accumulate toxin crystals [10]. Presently, information about the expression control of toxin was rare. Notably, El-Bendary et al. [23] identified two genes involved in sporulation, spo0A and spoIIAC, which might control expression of the binary toxin genes. Our lab identified several sporulation-associated genes by transposonmediated insertional mutagenesis, which affected expression of binary toxin genes [24]. These studies indicated that crystal protein synthesis is dependent on initiation of sporulation in L. sphaericus. In this study, we found that ppx disruption compromised the production of binary toxins. Although the $B$. cereus $\Delta \mathrm{ppx}$ impaired sporulation efficiency [4], the impairment of sporulation in L. sphaericus $\Delta \mathrm{ppx}$ was not observed (result not shown). Possibly, polyP affected binary toxins production by other unknown reasons, which remain to be investigated.

In summary, we provide evidence that ppx mutation accumulated massive polyP, compromised resistance to high osmotic stress, high $\mathrm{pH}$ and high temperature, and impaired motility and biofilm formation, and toxin production.

\section{Acknowledgements}

We thank Shinichi Kato (Regene Tiss Inc.) for supplying polyP14, polyP60 and polyP130 kindly. This project were supported by NFSC grants (30800002 and 31272384) and Doctoral scientific research of Hubei University for nationalities (MY2015B024).

\section{Conflict of Interest}

The authors declare that they have no conflicts of interest.

\section{References}

1. Rao NN, Gómez-García MR, Kornberg A. Inorganic polyphosphate: essential for growth and survival. Annu Rev Biochem. 2009;78:605- 


\section{7. doi: 10.1146/annurev.biochem.77.083007.093039.}

2. Kornberg A, Rao NN, Ault-Riché D. Inorganic polyphosphate: a molecule of many functions. Annu Rev Biochem. 1999;68:89-125.

3. Akiyama M, Crooke E, Kornberg A. An exopolyphosphatase of Escherichia coli. The enzyme and its ppx gene in a polyphosphate operon. J Biol Chem. 1993;268(1):633-9.

4. Shi X, Rao NN, Kornberg A. Inorganic polyphosphate in Bacillus cereus motility, biofilm formation, and sporulation. Proc Natl Acad Sci U S A. 2004;101(49):17061-5.

5. Frenzel E, Letzel T, Scherer S, Ehling-Schulz M. Inhibition of cereulide toxin synthesis by emetic Bacillus cereus via long-chain polyphosphates. Appl Environ Microbiol. 2011;77(4):1475-82. doi: 10.1128/AEM.02259-10.

6. Maier SK, Scherer S, Loessner MJ. Long-chain polyphosphate causes cell lysis and inhibits Bacillus cereus septum formation, which is dependent on divalent cations. Appl Environ Microbiol. 1999;65(9):3942-9.

7. Doruk T, Avican U, Camci IY, Gedik ST. Overexpression of polyphosphate kinase gene (ppk) increases bioinsecticide production by Bacillus thuringiensis. Microbiol Res. 2013;168(4):199-203. doi: 10.1016/j.micres.2012.11.009.

8. Baumann P, Unterman BM, Baumann L, Broadwell AH, Abbene SI Bowditch RD. Purification of the larvicidal toxin of Bacillus sphaericus and evidence for high-molecular-weight precursors. J Bacteriol. 1985;163(2):738-47

9. Broadwell AH, Linda Baumann, Paul Baumann. Larvicidal properties of the 42 and 51 Kilodalton Bacillus sphaericus proteins expressed in different Bacterial hosts: evidence for a binary toxin. Current Microbiology. 1990;21(6): 361-366.

10. Berry C. The bacterium, Lysinibacillus sphaericus, as an insect pathogen. J Invertebr Pathol. 2012;109(1):1-10. doi: 10.1016/j jip.2011.11.008.

11. Opota O, Gauthier NC, Doye A, Berry C, Gounon P, Lemichez E, et al. Bacillus sphaericus binary toxin elicits host cell autophagy as a response to intoxication. PLoS One. 2011;6(2):e14682. doi: 10.1371/ journal.pone.0014682.

12. Shi T, Ge Y, Zhao N, Hu X, Yuan Z. Polyphosphate kinase of Lysinibacillus sphaericus and its effects on accumulation of polyphosphate and bacterial growth. Microbiol Res. 2015;172:41-7. doi: 10.1016/j. micres.2014.12.002.
13. Schnepf E, Crickmore N, Van Rie J, Lereclus D, Baum J, Feitelson J, et al. Bacillus thuringiensis and its pesticidal crystal proteins. Microbiol Mol Biol Rev. 1998;62(3):775-806.

14. Yuan Z, Zhang Y, Liu E. Efficacy of Bacillus sphaericus C3-41 against Culex quinquefasciatus and its recycling in cadavers. Acta Entomologica Sinica. 1994;37(4):404-410.

15. Vallet-Gely I, Lemaitre B, Boccard F. Bacterial strategies to overcome insect defences. Nat Rev Microbiol. 2008;6(4):302-13. doi: 10.1038/ nrmicro1870

16. Brown MR, Kornberg A. The long and short of it - polyphosphate, PPK and bacterial survival. Trends Biochem Sci. 2008;33(6):284-90. doi: 10.1016/j.tibs.2008.04.005.

17. Kalfon A, Charles JF, Bourgouin C, de Barjac H. Sporulation of Bacillus sphaericus 2297: an electron microscope study of crystal-like inclusion biogenesis and toxicity to mosquito larvae. I Gen Microbiol. 1984;130(4):893-900.

18. Ault-Riché D, Fraley CD, Tzeng CM, Kornberg A. Novel assay reveals multiple pathways regulating stress-induced accumulations of inorganic polyphosphate in Escherichia coli. J Bacteriol. 1998;180(7):1841-7.

19. Alcántara C, Blasco A, Zúñiga M, Monedero V. Accumulation of polyphosphate in Lactobacillus spp. and its involvement in stress resistance. Appl Environ Microbiol. 2014;80(5):1650-9. doi: 10.1128/ AEM.03997-13.

20. Rashid MH, Rumbaugh K, Passador L, Davies DG, Hamood AN, Iglewski $\mathrm{BH}$, et al. Polyphosphate kinase is essential for biofilm development, quorum sensing, and virulence of Pseudomonas aeruginosa. Proc Natl Acad Sci U S A. 2000;97(17):9636-41.

21. Yang Y, Wang L, Gaviria A, Yuan Z, Berry C. Proteolytic stability of insecticidal toxins expressed in recombinant Bacilli. Appl Environ Microbiol. 2007;73(1):218-25

22. Davey ME, O'toole GA. Microbial biofilms: from ecology to molecular genetics. Microbiol Mol Biol Rev. 2000;64(4):847-67.

23. El-Bendary M, Priest FG, Charles JF, Mitchell WJ. Crystal protein synthesis is dependent on early sporulation gene expression in Bacillus sphaericus. FEMS Microbiol Lett. 2005;252(1):51-6.

24. Wu Y, Hu X, Ge Y, Zheng D, Yuan Z. Generation of mariner-based transposon insertion mutant library of Bacillus sphaericus 2297 and investigation of genes involved in sporulation and mosquito-larvicidal crystal protein synthesis. FEMS Microbiol Lett. 2012;330(2):105-12. doi: 10.1111/j.1574-6968.2012.02539.x. 